\title{
Aptitude à la coagulation de poudres de laits préacidifiés à $0^{\circ} \mathrm{C}$
}

\author{
par \\ R. EHSANI, M. BENNASAR et B. TARODO DE LA FUENTE*
}

\section{Ré s u m é}

Différents types de laits concentrés sont acidifiés par voie chimique (acide lactique) à basse température pour éviter leur floculation et ensuite séchés.

L'influence du mode de concentration (évaporation ou ultrafiltration), de l'addition de calcium et de présure, du taux de reconstitution et de la température de réchauffement sur les caractéristiques physico-chimiques des poudres de laits préacidifiés, leur aptitude à la coagulation, la texture et la composition des caillés correspondants, est étudiée.

Les produits obtenus présentent des avantages économiques et nutritionnels et des qualités proches de celles des fromages du commerce fabriqués par acidification directe du lait.

Mots clés

Laits secs - Coagulation - Caillé lactique.

Titre abrégé

Coagulation de laits secs préacidifiés à $0^{\circ} \mathrm{C}$.

\section{S u m m a ry}

EFFECT OF COLD ACIDIFICATION OF MILK BEFORE DRYING

ON THE COAGULATION ABILITY OF RECONSTITUTED PRODUCTS

Different kinds of concentrated milk are acidified at $0^{\circ} \mathrm{C}$ by use of chemical compounds (lactic acid) and subsequently dried; the

* Laboratoire de Technologie Alimentaire, Université des Sciences et Techniques du Languedoc, place Eugène-Bataillon - 34060 Montpellier cedex. 
acidification stape is done at low temperature to avoid flocculation. The effect of the concentration type (evaporation or ultrafiltration), of calcium and rennet addition, of reconstitution rate and heating on the physical and chemical properties of the preacidified milk powder, their coagulation ability and the corresponding curd texture and composition has been investigated.

Increasing reconstitution rate of evaporated concentrated milk result in a better cheese yield. With ultrafiltrated concentrated milk this increase is obtained also by calcium and rennet addition.

The resulting products have several economic and nutritional advantages and comparable properties with the commercial cheese obtained from direct acidified milk.

Key words

Dried milk - Coagulation - Lactic curd.

\section{INTRODUCTION}

L'explosion démographique dans les pays du Tiers-Monde ces dernières décennies jointe à l'augmentation de la consommation "per capita» des matières alimentaires protéiques et à l'insuffisance des ressources locales, et la surproduction des protéines dans les pays développés ont conduit les chercheurs et les organisations internationales à étudier des solutions propres à résoudre le problème de l'utilisation rationnelle de ces produits.

Parmi ceux-ci, la poudre de lait, qui possède une haute valeur nutritionnelle et qui présente les avantages de pouvoir être stockée, transportée et commercialisée facilement et rapidement, a acquis une place importante grâce en particulier à la variété de produits qu'elle permet d'obtenir. Les recherches entreprises dans ce domaine sur la production de caillé par acidification directe à partir de lait frais ou reconstitué ont conduit très tôt à la mise au point des méthodes de fabrication de différents types de fromages par de nombreux auteurs parmi lesquels on peut citer : Breene et al., 1964 ; Ernstrom, 1964 et 1965; Shehata et Olson, 1966; Little, 1967 et Larson et al., 1970.

Ce procédé présente de nombreux avantages par rapport à la fermentation lactique classique tels que : la simplicité des équipements nécessaires, la diminution des temps et des coûts de mise en œuvre, l'uniformité des produits finis et la possibilité de contrôler facilement la fabrication.

Par ailleurs, les travaux de Fox et Ernstrom, 1969, ont montré qu'un refroidissement préalable ralentissait et limitait la solubilisation du calcium au cours de l'acidification du lait et qu'il était pos- 
sible, dans ces conditions, de parvenir à un $\mathrm{pH}$ de 4,6 sans que la caséine précipite.

Cette technique est actuellement employée pour la fabrication continue du "Cottage Cheese » (Direct acid curd) et nous l'avons utilisée pour la fabrication de laits secs préacidifiés.

Nos travaux ont porté sur le séchage par atomisation de lait écrémé concentré et acidifié à $0^{\circ} \mathrm{C}$ par addition d'acide lactique jusqu'à pH 4,6. Les laits préacidifiés secs que nous avons obtenus donnent, après reconstitution et réchauffement, des caillés acides semblables à ceux que l'on obtient par la méthode de Fox et Ernstrom, 1969.

Nous avons étudié le rôle de plusieurs facteurs tels que le mode de concentration du lait initial (évaporation ou ultrafiltration), l'addition de calcium et de présure avant séchage, le taux de reconstitution et la température de coagulation sur les caractéristiques des poudres obtenues ou des caillés qu'elles permettent de préparer.

\section{MATERIELS ET METHODES}

\section{Types de laits}

Nous avons utilisé : soit des laits de mélange écrémés et pasteurisés, soit des laits reconstitués à partir de poudre Spray écrémée de qualité bas chauffage.

Les laits refroidis à $0^{\circ} \mathrm{C}$ sont acidifiés par l'acide lactique $2 \mathrm{~N}$ à la même température. L'addition d'acide se fait lentement sous forte agitation et le mélange est laissé au repos pendant $40 \mathrm{~min}$ suivant les instructions de Ernstrom, 1964 et 1965. Le but recherché étant l'obtention d'un coagulum à un $\mathrm{pH}$ voisin de 4,6 quel que soit le taux de reconstitution, bien que le $\mathrm{pH}$ isoélectrique des caséines varie légèrement avec la concentration du lait comme l'ont montré Alais et Tarodo de la Fuente, 1970, nous avons préféré acidifier systématiquement les laits de départ jusqu'au $\mathrm{pH}$ isoélectrique des caséines dans un lait normal $(4,6)$.

Certains laits sont additionnés, à température ambiante et après concentration, de chlorure de calcium à la dose de 6 millimoles de calcium par litre de lait initial et emprésurés à l'aide d'une solution de force 10000 obtenue à partir d'une présure animale en poudre (Maret) de force initiale 275 U.P. (Unité Présure)/g selon Berridge, 1952 ; d'autres sont seulement emprésurés. Les conditions d'emprésurage sont voisines de celles employées pour la fabrication industrielle des pâtes fraîches : le lait après acidification est emprésuré à la dose de $5 \mathrm{ml}$ de la solution précitée pour 1001 de concentré et laissé avant séchage une douzaine d'heures à basse température pour permettre l'action partielle de l'enzyme. 
L'étude des divers paramètres a été effectuée sur ces types de laits reconstitués à des taux variables. Nous ne donnerons dans ce travail que les résultats les plus représentatifs et correspondant au taux de reconstitution pour lequel toutes les catégories de laits définies ci-dessus ont été testées. En outre, les valeurs figurant dans chaque tableau résultent parfois d'expériences séparées dans le temps et ne présentent pas forcément de concordance directe entre elles. Ce point sera précisé dans le texte.

\section{Traitements technologiques}

La concentration des laits est effectuée :

- Soit par évaporation (EV) sous vide jusqu'à un facteur de concentration de quatre fois avec un appareil à plaques A.P.V. "Junior ", dans des conditions telles que la température du produit ne dépasse pas $50^{\circ} \mathrm{C}$ et que la durée de l'opération soit d'une heure environ.

- Soit par ultrafiltration (UF) jusqu'à un facteur 3, dans un appareil Rhône-Poulenc "Pléiade 》 UF P7 équipé de membranes IRIS 3042 ayant un " cut off » de 20000 . Dans ce cas, l'opération conduite à $18^{\circ} \mathrm{C}$ pouvant demander plusieurs heures, le lait est additionné de $0,5 \mathrm{ml}$ de toluène par litre pour limiter les risques de fermentation.

Le séchage des laits concentrés acidifiés maintenus à $0^{\circ} \mathrm{C}$ est effectué dans une tour d'atomisation Anhydro «Lab 3 ». La température de l'air chaud est de $160^{\circ} \mathrm{C}$ et celle de la poudre ne dépasse pas $90^{\circ} \mathrm{C}$.

\section{Méthodes d'analyses}

La matière sèche (MS) des laits, des caillés et des sérums et l'humidité des poudres sont déterminées à l'étuve à $100 \pm 2^{\circ} \mathrm{C}$.

L'azote total (N) est mesuré par la méthode de Kjeldahl selon Alais, 1962, et les matières azotées totales (MAT) correspondantes sont calculées à l'aide du facteur 6,38 d'après Gordon and Kalan, 1974.

Le calcium (Ca) est évalué par spectrophotométrie d'absorption atomique à l'aide d'un appareil Varian "Techtron » AA 6 suivant Linden, 1971, et le calcium soluble est dosé par la même technique après ultrafiltration du lait d'après Davies et White, 1960.

Le $\mathrm{pH}$ est mesuré à l'aide d'un $\mathrm{pH}$ mètre Methrohm « Hérisau " E 300 B.

L'aptitude à la coagulation des laits secs préacidifiés reconstitués est étudiée par la méthode thrombélastographique appliquée au lait par Frentz, 1965, et Tarodo de la Fuente et al., 1969, à l'aide d'un appareil Hellige « $\mathrm{C}$ » modifié. Le lait reconstitué à $0^{\circ} \mathrm{C}$ est placé dans la cuve de mesure maintenue à la même température et l'enre- 
gistrement est mis en route en même temps que le réchauffement de la cuve.

Dans ces conditions, le paramètre $r$ qui représente le temps écoulé entre le début du réchauffement du lait et le dédoublement du tracé est appelé temps de floculation et Tf est la température du lait à ce moment-là. Le paramètre $K$ qui caractérise la vitesse de raffermissement du coagulum est mesuré lorsque l'écartement du tracé atteint $20 \mathrm{~mm}$ et Tc est la température correspondante.

La fermeté des gels est mesurée avec un pénétromètre automatique Herzog, et exprimée par l'indice de fermeté If $=1000$ /A défini par Tarodo de la Fuente et al., 1971, dans lequel A représente l'enfoncement en $\mathrm{mm}$ du plongeur.

La vitesse d'égouttage est évaluée par mesure du temps nécessaire ( $\mathrm{t}$ ) pour recueillir $50 \%$ du volume total (v) de sérum exsudé par filtration à température ambiante pendant $90 \mathrm{~min}$ selon la méthode de Lelièvre, 1977, à partir d'un coagulum maintenu pendant $1 \mathrm{~h}$ à des températures variables et provenant de $50 \mathrm{ml}$ de lait préacidifié reconstitué à $0^{\circ} \mathrm{C}$ à différentes teneurs en matières sèches.

Compte tenu de la difficulté de mesurer directement la fermeté des caillés, celle-ci est appréciée après égouttage en calculant le rapport des extraits secs ( $\mathrm{Re}$ ) des caillés et des sérums correspondants auquel elle est proportionnelle comme l'ont montré Tarodo de la Fuente et al., 1971.

Le rendement fromager (Rf) représente le nombre de grammes de caillé obtenu à partir de $100 \mathrm{ml}$ de lait préacidifié reconstitué.

\section{RESULTATS}

\section{Effet du refroidissement sur la fixation de calcium sur les protéines de lait acidifié}

Un lait reconstitué à $10 \%$ à partir de poudre est fractionné en 3 échantillons $\mathrm{A}, \mathrm{B}$ et $\mathrm{C}$ qui sont acidifiés jusqu'à $\mathrm{pH}$ 4,6 à différentes températures.

Après un temps d'attente suffisant à la formation éventuelle d'un gel, les échantillons sont brassés avec addition de calcium pour certains et laissés ensuite au repos pendant $5 \mathrm{~h}$ à $0^{\circ} \mathrm{C}$. Les conditions opératoires et les caractéristiques physiques des produits obtenus ainsi que la teneur en calcium fixée sur les protéines sont consignées dans le tableau 1.

Nous observons que seuls les laits $\mathrm{A}$ et $\mathrm{B}$ acidifiés à température ambiante $\left(25^{\circ} \mathrm{C}\right)$ donnent lieu à la formation d'un véritable gel. Ces mêmes laits après refroidissement et agitation énergique 
TABLEAU 1 - TABLE 1

Conditions d'acidification et caractéristiques physico-chimiques des produits obtenus après agitation

Acidification conditions and physico-chemical caracteristics of products obtained after agitation

\begin{tabular}{|c|c|c|c|c|c|c|c|c|c|c|}
\hline \multirow{2}{*}{ Types de lait } & \multicolumn{3}{|c|}{ Laits reconstitués } & \multirow{2}{*}{$\begin{array}{l}\text { Température } \\
\text { acidification } \\
{ }^{\circ} \mathrm{C}\end{array}$} & \multirow{2}{*}{ Gel } & \multirow{2}{*}{$\begin{array}{c}\text { Température } \\
\text { agitation } \\
{ }^{\circ} \mathrm{C}\end{array}$} & \multirow{2}{*}{$\begin{array}{c}\text { Addition Ca } \\
\text { mmoles/1 }\end{array}$} & \multirow{2}{*}{$\begin{array}{l}\text { Température } \\
\text { repos }(5 \mathrm{~h}) \\
{ }^{\circ} \mathrm{C}\end{array}$} & \multirow{2}{*}{$\begin{array}{l}\text { Aspect } \\
\text { après } \\
\text { repos }\end{array}$} & \multirow{2}{*}{$\begin{array}{c}\text { Ca fixé } \\
\%\end{array}$} \\
\hline & $\mathrm{pH}$ & $\begin{array}{l}\mathrm{Ca} \\
\mathrm{g} / 1\end{array}$ & $\begin{array}{c}\text { Mat } \\
\mathrm{g} / \mathrm{l}\end{array}$ & & & & & & & \\
\hline A & 6,6 & 1,28 & 32,6 & 25 & oui & 0 & 0 & 0 & très épais & 8,6 \\
\hline B & $"$ & $»$ & $»$ & 25 & oui & 0 & 6 & 0 & épais & 9,5 \\
\hline C & $"$ & $»$ & $"$ & 0 & non & 0 & 0 & 0 & fluide & 13,9 \\
\hline
\end{tabular}






fig. 1

Courbes d'acidification

ㅁ: lait écrémé reconstitué $(90 \mathrm{~g} / \mathrm{l})$.

- : lait concentré par ultrafiltration (3 fois).

- : lait concentré par évaporation (4 fois).

Acidification curve

ㅁ: reconstituted skim milk $(90 \mathrm{~g} / \mathrm{l})$.

- : ultrafiltrated concentrated milk (3-fold).

- : evaporated concentrated milk (4-fold). 
retrouvent une texture fluide quoique plus épaisse que celle du lait $\mathrm{C}$ acidifié dès le départ à $0^{\circ} \mathrm{C}$.

La gélification par acidification s'avère donc réversible à basse température et s'accompagne de la refixation sur les protéines d'une partie du calcium solubilisée qui reste malgré tout inférieure à celle du lait C. La quantité de calcium fixée est cependant supérieure dans le cas du lait $\mathrm{B}$ enrichi en ce sel et conduit à une meilleure fluidisation.

\section{Acidification des différents types de laits}

L'évolution du $\mathrm{pH}$ en fonction de la quantité d'acide lactique ajoutée à différents types de laits écrémés : reconstitué normal, concentré par ultrafiltration ou évaporation est représentée sur la figure 1 .

Le $\mathrm{pH}$ des laits de départ est de 6,6 pour le lait reconstitué normal et celui concentré par ultrafiltration, et de 6,4 environ pour le lait concentré par évaporation.

Nous constatons que l'acidité augmente très rapidement au début et plus lentement ensuite et que le volume d'acide nécessaire pour atteindre un $\mathrm{pH}$ de 4,6 croît avec la concentration en matières sèches pour une même prise d'essai de lait.

Cependant cette même quantité d'acide exprimée par rapport aux matières azotées diminue d'autant plus que celles-ci augmentent. C'est ainsi que si l'on prend comme référence 1 pour la quantité d'acide nécessaire par rapport aux matières azotées dans le cas du lait normal témoin, cette quantité devient 0,94 pour le lait concentré 3 fois par ultrafiltration et 0,92 pour celui concentré 4 fois par évaporation.

Il faut noter également que les laits enrichis en calcium subissent une baisse du $\mathrm{pH}$ initial qui réduit d'autant le volume d'acide nécessaire pour atteindre le $\mathrm{pH}$ de 4,6 .

\section{Caractéristiques des laits préacidifiés secs}

Les caractéristiques des différents types de laits concentrés, préacidifiés jusqu'à pH 4,6, additionnés ou non de calcium et de présure et soumis au séchage ainsi que des poudres correspondantes sont indiquées dans le tableau 2.

Nous constatons que les poudres issues des laits concentrés non enrichis en calcium et présure sont plus humides. Le rapport $\mathrm{Ca} / \mathrm{N}$ est plus faible pour celles qui proviennent des laits concentrés par ultrafiltration et il augmente évidemment quel que soit le type de lait initial lorsque celui-ci est additionné de calcium. La présure ne semble pas avoir d'influence sur les taux de matières azotées et de calcium. 
$T A B L E A U 2-T A B L E 2$

Caractéristiques des laits concentrés préacidifiés, des poudres et des laits reconstitués correspondants

Caracteristics of preacidified concentrated milks, powders and reconstituted milks

\begin{tabular}{|c|c|c|c|c|c|c|c|c|c|c|}
\hline \multirow[b]{2}{*}{$\mathrm{N}^{\circ}$} & \multicolumn{2}{|c|}{ Laits de fabrication } & \multicolumn{2}{|c|}{ Addition avant séchage } & \multicolumn{4}{|c|}{ Laits secs } & \multicolumn{2}{|c|}{ Laits reconstitués } \\
\hline & $\begin{array}{l}\text { Mode de } \\
\text { concentra- } \\
\text { tion }\end{array}$ & $\begin{array}{c}\mathrm{M} \mathrm{S} \\
\mathrm{g} / \mathrm{l}\end{array}$ & $\begin{array}{c}\mathrm{Ca} \\
\mathrm{mmoles} / 1\end{array}$ & $\begin{array}{l}\text { Présure } \\
\mathrm{ml} / 100 \mathrm{l}\end{array}$ & $\frac{\mathrm{N}}{\mathrm{mg} / \mathrm{g}} \mathrm{MS}$ & $\begin{array}{c}\mathrm{Ca} \\
\mathrm{mg} / \mathrm{g} \mathrm{MS}\end{array}$ & $\mathrm{Ca} / \mathrm{N}$ & $\underset{\%}{\mathrm{H}_{2} \mathrm{O}}$ & $\mathrm{pH}$ & $\begin{array}{c}\text { Ca fixé } \\
\%\end{array}$ \\
\hline 1 & E V & 388,4 & 0 & 0 & 51,2 & 14,0 & 0,273 & 8,9 & 4,85 & 16,2 \\
\hline 2 & E V & $»$ & 24 & 0 & 51,4 & 16,1 & 0,313 & 4,9 & 4,90 & 18,1 \\
\hline 3 & E V & $»$ & 24 & 5 & 51,4 & 16,0 & 0,311 & 5,8 & 4,90 & 17,8 \\
\hline 4 & $\mathrm{U} F$ & 136,0 & 0 & 0 & 79,9 & 16,3 & 0,204 & 10,7 & 4,70 & 14,2 \\
\hline 5 & U F & $》$ & 18 & 0 & 79,8 & 17,4 & 0,218 & 6,1 & 4,78 & 14,8 \\
\hline 6 & U F & » & 18 & 5 & 79,9 & 17,3 & 0,217 & 6,4 & 4,80 & 14,3 \\
\hline
\end{tabular}




\section{Caractéristiques des laits préacidifiés reconstitués}

Les valeurs du $\mathrm{pH}$ et du taux de calcium fixé sur les protéines des différents types de laits après reconstitution à $0^{\circ} \quad \mathrm{C}$ et à $8,5 \%$ de matières sèches sont indiquées dans le tableau 2. Nous observons que ces valeurs sont en général plus élevées pour les laits concentrés initialement par évaporation.

L'addition de calcium s'accompagne d'une diminution de l'acidité et entraîne une meilleure fixation de ce sel sur les protéines dans tous les cas, tandis que l'emprésurage ne semble avoir que peu d'effet.

L'augmentation de la concentration en matières sèches des laits reconstitués se traduit par une fixation accrue de calcium sur les protéines, malgré une diminution parallèle du $\mathrm{pH}$ comme nous le voyons dans le tableau 3.

TABLEAU 3 - TABLE 3

Influence du taux de reconstitution sur les caractéristiques des différents types de laits préacidifiés reconstitués

Influence of the reconstitution rate on caracteristics of preacidified reconstituted milks

\begin{tabular}{|c|c|c|c|c|c|c|c|c|}
\hline Laits fabrication* & \multicolumn{4}{|c|}{1} & \multicolumn{4}{|c|}{4} \\
\hline Taux reconstitution $\%$ & 8,5 & 12,5 & 16,0 & 23,0 & 8,5 & 12,5 & 16,0 & 23,0 \\
\hline $\mathrm{pH}$ & 4,85 & 4,75 & 4,71 & 4,66 & 4,70 & 4,62 & 4,59 & 4,56 \\
\hline Ca fixé \% & 15,9 & 17,6 & 18,7 & 20,4 & 15,6 & 17,2 & 17,9 & 18,5 \\
\hline
\end{tabular}

* Cf. : tableau 2 .

\section{Aptitude à la floculation et la coagulation des laits préacidifiés reconstitués}

L'aptitude à la floculation et à la coagulation des différents types de laits préacidifiés reconstitués à $0^{\circ} \mathrm{C}$ et à $16 \%$ de matières sèches est évaluée par la méthode thrombélastographique (tab. 4). Nous constatons que l'addition de calcium se traduit par une augmentation des temps de floculation ( $\mathrm{r}$ ) et de raffermissement du coagulum (K) ainsi que des températures correspondantes (Tf, Tc). L'emprésurage entraîne par contre une nette diminution de tous ces facteurs qui reviennent à des valeurs proches ou inférieures à celles des laits témoins. Ces divers paramètres sont dans l'ensemble plus élevés dans le cas des laits concentrés par évaporation. L'influence du taux 
TABLEAU 4 - TABLE 4

Etude des paramètres de floculation et de coagulation des différents types de laits préacidifiés reconstitués

Flocculation and clotting parameters of preacidified reconstituted milks

\begin{tabular}{c|c|c|c|c}
\hline $\begin{array}{c}\text { Laits de } \\
\text { fabrication* }\end{array}$ & $\begin{array}{c}\mathrm{r} \\
\mathrm{min}\end{array}$ & $\begin{array}{c}\mathrm{Tf} \\
{ }^{\circ} \mathrm{C}\end{array}$ & $\begin{array}{c}\mathrm{K} \\
\mathrm{min}\end{array}$ & $\begin{array}{c}\mathrm{Tc} \\
{ }^{\circ} \mathrm{C}\end{array}$ \\
\cline { 2 - 3 } 1 & 32,0 & 15,5 & 18,0 & 21,0 \\
2 & 57,0 & 20,8 & 24,5 & 24,9 \\
3 & 30,0 & 14,9 & 11,0 & 17,0 \\
4 & 20,0 & 8,5 & 4,5 & 10,2 \\
5 & 36,0 & 16,5 & 5,5 & 18,0 \\
6 & 16,0 & 5,8 & 4,0 & 9,3 \\
\hline
\end{tabular}

* Cf. : tableau 2.

TABLEAU 5 - TABLE 5

Influence du taux de reconstitution sur les paramètres de floculation et de coagulation des différents types de laits préacidifiés reconstitués

Influence of the reconstitution rate on flocculation and clotting parameters of preacidified reconstituted milks

\begin{tabular}{|c|c|c|c|c|c|c|c|c|}
\hline Lait fabrication* & \multicolumn{4}{|c|}{1} & \multicolumn{4}{|c|}{4} \\
\hline Taux reconstitution $\%$ & 8,5 & 12,5 & 16,0 & 23,0 & 8,5 & 12,5 & 16,0 & 23,0 \\
\hline $\mathrm{r} \min$ & 11,5 & 25,6 & 37,0 & 38,0 & 11,0 & 22,0 & 27,0 & 28,0 \\
\hline $\mathrm{Tf}{ }^{\circ} \mathrm{C}$ & 8,0 & 12,6 & 15,5 & 15,7 & 6,0 & 9,8 & 14,0 & 14,2 \\
\hline $\mathrm{K} \min$ & 42,5 & 24,0 & 17,0 & 13,0 & 20,5 & 10,2 & 6,8 & 6,2 \\
\hline $\mathrm{Tc}{ }^{\circ} \mathrm{C}$ & 21,0 & 20,1 & 21,5 & 20,0 & 15,0 & 15,1 & 15,1 & 15,6 \\
\hline
\end{tabular}

* Cf. : tableau 2.

de reconstitution dont les résultats sont rassemblés dans le tableau 5 montre que la floculation se produit après un temps d'autant plus long et à une température d'autant plus élevée que la teneur en matières sèches augmente, avec cependant un tassement de ces valeurs au-delà de $16 \%$. 
Dans le même temps, nous notons une accélération de la vitesse de raffermissement du coagulum avec peu de variation de la température finale.

\section{Etude de la synérèse et de la texture des gels et des caillés des laits préacidifiés reconstitués}

Les différents types de laits sont reconstitués à $0^{\circ} \mathrm{C}$ et $12,5 \%$ de matières sèches et réchauffés à $40^{\circ} \mathrm{C}$. Les quantités de sérum (v) et le temps nécessaire pour en recueillir $50 \%$ (t) qui exprime la vitesse d'exsudation ainsi que les fermetés des gels (If) et des caillés $(\mathrm{Re})$ correspondants sont indiqués dans le tableau 6 .

\section{TABLEAU 6 - TABLE 6}

Etude de la synérèse et de la texture des gels et des caillés des différents types de laits préacidifiés reconstitués

Study of syneresis and gel and curd texture of preacidified reconstituted milks

\begin{tabular}{|c|c|c|c|c|c|}
\hline \multirow{2}{*}{$\begin{array}{l}\text { Laits de } \\
\text { fabrication* }\end{array}$} & \multirow{2}{*}{$\begin{array}{l}\mathrm{pH} \text { laits } \\
\text { reconstitués }\end{array}$} & \multicolumn{2}{|c|}{ Exsudation } & \multicolumn{2}{|c|}{ Fermeté } \\
\hline & & $t_{\min }$ & $\mathrm{vml}$ & $\begin{array}{c}\text { If } \\
\text { (coagulum) }\end{array}$ & $\begin{array}{c}\mathrm{Re} \\
\text { (caillé) }\end{array}$ \\
\hline 1 & 4,75 & 2,9 & 31,6 & 6,3 & 2,1 \\
\hline 2 & 4,80 & 4,2 & 33,9 & 6,5 & 2,2 \\
\hline 3 & 4,80 & 2,8 & 34,9 & 6,7 & 2,3 \\
\hline 4 & 4,64 & 4,1 & 25,3 & 9,2 & 2,6 \\
\hline 5 & 4,68 & 4,8 & 24,8 & 9,2 & 2,5 \\
\hline 6 & 4,68 & 3,5 & 26,0 & 9,3 & 3,1 \\
\hline
\end{tabular}

* Cf. : tableau 2.

Nous constatons que les laits concentrés par évaporation conduisent à un égouttage plus rapide et plus important malgré une acidité moins forte, mais que les coagulums et les caillés sont moins fermes que ceux provenant des laits concentrés par ultrafiltration. L'enrichissement en calcium qui provoque un ralentissement de l'égouttage et une légère augmentation du volume de sérum surtout dans le cas des laits évaporés, ne semble avoir que peu d'effet sur la texture des coagulums et des gels.

L'emprésurage par contre entraîne dans tous les cas une synérèse accélérée et poussée qui se traduit par une meilleure fermeté des gels et des caillés. 
Ces mêmes paramètres étudiés en fonction du taux de reconstitution dans le tableau 7 montrent que lorsque celui-ci augmente, la vitesse d'égouttage et la quantité totale de sérum chutent malgré la diminution du $\mathrm{pH}$, tandis que la fermeté des gels s'accroît mais que celle des caillés égouttés baisse.

\section{TABLEAU 7 - TABLE 7}

Influence du taux de reconstitution sur la synérèse et la texture des gels et des caillés des laits préacidifiés reconstitués

Influence of the reconstitution rate on syneresis and gel and curd texture of preacidified reconstituted milks

\begin{tabular}{|c|c|c|c|c|c|c|c|c|}
\hline \multirow{2}{*}{ Laits fabrication* } & \multicolumn{4}{|c|}{1} & \multicolumn{4}{|c|}{4} \\
\hline & 8,5 & 12,5 & 16,0 & 23,0 & 8,5 & 12,5 & 16,0 & 23,0 \\
\hline $\mathrm{pH}$ & 4,85 & 4,75 & 4,71 & 4,65 & 4,74 & 4,64 & 4,60 & 4,57 \\
\hline $\mathrm{t} \min$ & 1,6 & 2,9 & 4,9 & 10,0 & 1,5 & 4,1 & 8,6 & 18,0 \\
\hline $\mathrm{v} \mathrm{ml}$ & 38,6 & 31,6 & 26,7 & 17,3 & 34,5 & 25,3 & 20,6 & 7,5 \\
\hline If & n.m.** & 6,3 & 7,3 & 9,4 & n.m.** & 9,2 & 11,1 & 14,6 \\
\hline $\mathrm{Re}$ & 2,7 & 2,1 & 1,8 & 1,5 & 3,4 & 2,6 & 2,2 & 1,7 \\
\hline $\begin{array}{l}* \text { Cf. : tableau } 2 . \\
* \text { Non mesurable. }\end{array}$ & & & & & & & & \\
\hline
\end{tabular}

Les valeurs du tableau 8 illustrent l'influence de l'élévation de la température de réchauffement qui accélère l'égouttage et a tendance à augmenter la quantité de sérum exsudé ainsi que la fermeté des gels et des caillés quel que soit le type de lait.

\section{Rendements et composition des caillés et des sérums des laits préacidifiés reconstitués}

Les compositions en matières sèches (MS), matières azotées totales (MAT) et calcium (Ca) des sérums et des caillés, ainsi que les rendements fromager ( $\mathrm{Rf})$, en azote $(\mathrm{N})$ et en calcium $(\mathrm{Ca})$ des caillés issus des différents types de laits préacidifiés reconstitués dans les conditions précédemment décrites (cf. § III 6) sont indiqués dans le tableau 9.

Dans l'ensemble les caillés de laits concentrés par ultrafiltration sont plus riches en matières sèches, matières azotées et calcium que ceux provenant des laits évaporés. 


\section{$T A B L E A U 8-T A B L E 8$}

Influence de la température de réchauffement sur la synérèse et la texture des gels et des caillés des laits préacidifiés reconstitués

Influence of the heating temperature on syneresis and gel and curd texture of preacidified reconstituted milks

\begin{tabular}{|c|c|c|c|c|c|c|}
\hline Laits de fabrication* & \multicolumn{3}{|c|}{1} & \multicolumn{3}{|c|}{4} \\
\hline Température réchauffement ${ }^{\circ} \mathrm{C}$ & 25 & 32 & 40 & 25 & 32 & 40 \\
\hline $\mathrm{t} \min$ & 6,2 & 3,6 & 2,9 & 15,6 & 6,6 & 4,1 \\
\hline $\mathrm{v} \mathrm{ml}$ & 31,1 & 31,4 & 31,6 & 21,8 & 25,2 & 25,3 \\
\hline If & 6,0 & 6,2 & 6,3 & 8,4 & 8,9 & 9,2 \\
\hline $\mathrm{Re}$ & 1,9 & 2,0 & 2,1 & 2,4 & 2,5 & 2,6 \\
\hline
\end{tabular}

* Cf. : tableau 2.

Les sérums des laits ultrafiltrés contiennent également plus de protéines et de calcium mais sont moins concentrés en matières sèches. L'addition de calcium entraîne une perte un peu plus importante de tous les éléments dans les sérums mais son action au niveau des caillés est très différente suivant le mode de concentration du lait initial ; lorsque le lait est concentré par évaporation, nous observons une hausse des taux des diverses substances mesurées ainsi que du rapport $\mathrm{Ca} / \mathrm{N}$ tandis que les rendements baissent légèrement ; dans le cas du lait concentré par ultrafiltration, seule la teneur en calcium du caillé augmente et donc le rapport $\mathrm{Ca} / \mathrm{N}$ et par contre les divers rendements ont tendance à s'élever.

L'emprésurage n'a pratiquement aucun effet sur la composition du sérum, il se traduit dans les caillés des différents laits par une élévation des taux de matières sèches et d'azote, une diminution $\mathrm{du}$ calcium et donc du rapport $\mathrm{Ca} / \mathrm{N}$ et une chute des divers rendements. L'accroissement du taux de reconstitution (tab. 10) se traduit par une meilleure rétention des différents éléments dans le caillé, à l'exception des matières azotées, avec une amélioration de l'ensemble des rendements pour les deux catégories de lait.

L'étude de la température de réchauffement avec des laits reconstitués à $8,5 \%$ de matières sèches, nous a donné les valeurs figurant dans le tableau 11.

La variation de cette température a peu d'effets significatifs sur le lait concentré par évaporation, si ce n'est une légère amélioration du taux de matières sèches du caillé lorsque le chauffage est plus 
TABLEAU 9 - TABLE 9

Composition des sérums et des caillés des différents types de laits préacidifiés reconstitués Whey and curd composition of preacidified reconstituted milks

\begin{tabular}{|c|c|c|c|c|c|c|c|c|c|c|}
\hline \multirow{2}{*}{$\begin{array}{l}\text { Laits de } \\
\text { fabrication* }\end{array}$} & \multicolumn{3}{|c|}{ Sérum (composition) } & \multicolumn{4}{|c|}{ Caillé (composition) } & \multicolumn{3}{|c|}{ Caillé (rendement) } \\
\hline & $\begin{array}{l}\text { MS } \\
\mathrm{g} / 1\end{array}$ & $\begin{array}{c}\mathrm{MAT} \\
\mathrm{g} / 1\end{array}$ & $\begin{array}{l}\mathrm{Ca} \\
\mathrm{g} / \mathrm{l}\end{array}$ & $\begin{array}{c}\mathrm{MS} \\
\mathrm{g} / \mathrm{kg}\end{array}$ & $\begin{array}{l}\mathrm{MAT} \\
\mathrm{g} / \mathrm{kg}\end{array}$ & $\begin{array}{r}\mathrm{Ca} \\
\mathrm{g} / \mathrm{kg}\end{array}$ & $\mathrm{Ca} / \mathrm{N}$ & $\begin{array}{l}\mathrm{Rf} \\
\%\end{array}$ & $\begin{array}{l}\mathrm{N} \\
\%\end{array}$ & $\begin{array}{l}\mathrm{Ca} \\
\%\end{array}$ \\
\hline 1 & 95,8 & 9,3 & 1,67 & 193,0 & 101,3 & 1,76 & 0,111 & 32,4 & 84,4 & 34,5 \\
\hline 2 & 96,9 & 9,5 & 1,83 & 205,0 & 117,3 & 2,49 & 0,136 & 28,2 & 83,3 & 32,9 \\
\hline 3 & 96,8 & 9,6 & 1,85 & 213,0 & 125,9 & 2,33 & 0,118 & 26,2 & 82,7 & 31,5 \\
\hline 4 & 78,1 & 11,9 & 2,40 & 225,1 & 168,3 & 2,39 & 0,091 & 30,4 & 89,6 & 34,5 \\
\hline 5 & 80,1 & 13,3 & 2,68 & 203,0 & 155,0 & 3,15 & 0,130 & 40,4 & 90,0 & 47,4 \\
\hline 6 & 80,2 & 13,6 & 2,67 & 237,0 & 160,0 & 2,61 & 0,104 & 34,2 & 88,0 & 35,5 \\
\hline
\end{tabular}

* Cf. : tableau 2. 
TABLEAU $10-T A B L E 10$

Influence du taux de reconstitution sur la composition des sérums et des caillés des différents types de laits préacidifiés reconstitués

Influence of the reconstitution rate on whey and curd composition of preacidified reconstituted milks

\begin{tabular}{|c|c|c|c|c|c|c|c|c|c|c|c|}
\hline \multirow{2}{*}{$\begin{array}{l}\text { Laits de } \\
\text { fabrication* }\end{array}$} & \multirow{2}{*}{$\begin{array}{c}\text { Taux de } \\
\text { reconstitution } \\
\%\end{array}$} & \multicolumn{3}{|c|}{ Sérum (composition) } & \multicolumn{4}{|c|}{ Caillé (composition) } & \multicolumn{3}{|c|}{ Caillé (rendement) } \\
\hline & & $\begin{array}{l}\text { MS } \\
\mathrm{g} / \mathrm{l}\end{array}$ & $\underset{\mathrm{g} / \mathrm{l}}{\mathrm{MAT}}$ & $\begin{array}{l}\mathrm{Ca} \\
\mathrm{g} / \mathrm{l}\end{array}$ & $\underset{\mathrm{g} / \mathrm{kg}}{\mathrm{MS}}$ & $\begin{array}{l}\text { MAT } \\
\mathrm{g} / \mathrm{kg}\end{array}$ & $\underset{\mathrm{g} / \mathrm{kg}}{\mathrm{Ca}}$ & $\mathrm{Ca} / \mathrm{N}$ & $\begin{array}{l}\mathrm{Rf} \\
\%\end{array}$ & $\begin{array}{l}\mathrm{N} \\
\%\end{array}$ & $\begin{array}{l}\mathrm{Ca} \\
\%\end{array}$ \\
\hline \multirow{4}{*}{1} & 8,5 & 65,1 & 6,0 & 1,13 & 172,6 & 106,3 & 1,18 & 0,071 & 20,7 & 82,2 & 21,5 \\
\hline & 12,5 & 95,8 & 9,3 & 1,67 & 193,0 & 101,3 & 1,76 & 0,111 & 32,4 & 84,4 & 34,5 \\
\hline & 16 & 125,0 & 12,4 & 2,13 & 216,0 & 104,2 & 2,21 & 0,135 & 42,4 & 86,6 & 44,2 \\
\hline & 23 & 181,9 & 18,3 & 3,01 & 262,1 & 106,2 & 3,18 & 0,194 & 59,7 & 90,5 & 63,3 \\
\hline \multirow{4}{*}{4} & 8,5 & 49,2 & 8,9 & 1,90 & 215,1 & 161,4 & 1,16 & 0,046 & 23,5 & 85,2 & 16,3 \\
\hline & 12,5 & 78,1 & 11,9 & 2,40 & 225,1 & 168,3 & 2,39 & 0,091 & 30,4 & 89,6 & 34,5 \\
\hline & 16 & 94,8 & 15,7 & 2,98 & 234,0 & 161,6 & 2,69 & 0,106 & 40,3 & 90,9 & 41,3 \\
\hline & 23 & 149,1 & 24,1 & 4,62 & 273,9 & 161,5 & 3,69 & 0,146 & 62,7 & 96,0 & 59,8 \\
\hline
\end{tabular}

* Cf. : tableau 2. 


\section{TABLEAU $11-T A B L E 11$}

Influence de la température de réchauffement sur la composition des sérums et des caillés des différents types de laits préacidifiés reconstitués

Influence of heating temperature on whey and curd composition of preacidified reconstituted milks

\begin{tabular}{|c|c|c|c|c|c|c|c|c|c|c|c|}
\hline \multirow{2}{*}{$\begin{array}{l}\text { Laits de } \\
\text { fabrication* }\end{array}$} & \multirow{2}{*}{$\begin{array}{l}\text { Température } \\
\text { réchauffement } \\
{ }^{\circ} \mathrm{C}\end{array}$} & \multicolumn{3}{|c|}{ Sérum (composition) } & \multicolumn{4}{|c|}{ Caillé (composition) } & \multicolumn{3}{|c|}{ Caillé (rendement) } \\
\hline & & $\begin{array}{l}\mathrm{MS} \\
\mathrm{g} / 1\end{array}$ & $\begin{array}{c}\mathrm{MAT} \\
\mathrm{g} / \mathrm{l}\end{array}$ & $\begin{array}{l}\mathrm{Ca} \\
\mathrm{g} / 1\end{array}$ & $\begin{array}{l}\mathrm{MS} \\
\mathrm{g} / \mathrm{kg}\end{array}$ & $\begin{array}{l}\mathrm{MAT} \\
\mathrm{g} / \mathrm{kg}\end{array}$ & $\begin{array}{c}\mathrm{Ca} \\
\mathrm{g} / \mathrm{kg}\end{array}$ & $\mathrm{Ca} / \mathrm{N}$ & $\begin{array}{l}\mathrm{Rf} \\
\%\end{array}$ & $\begin{array}{l}\mathrm{N} \\
\%\end{array}$ & $\begin{array}{l}\mathrm{Ca} \\
\%\end{array}$ \\
\hline \multirow{3}{*}{1} & 25 & 64,7 & 5,8 & 1,15 & 163,5 & 107,2 & 1,21 & 0,072 & 21,7 & 83,7 & 22,7 \\
\hline & 32 & 64,5 & 5,8 & 1,14 & 169,3 & 105,8 & 1,17 & 0,071 & 20,8 & 82,9 & 21,4 \\
\hline & 40 & 65,1 & 6,0 & 1,13 & 172,6 & 106,3 & 1,18 & 0,071 & 20,7 & 82,2 & 21,5 \\
\hline \multirow{3}{*}{4} & 25 & 49,3 & 9,0 & 1,84 & 200,5 & 138,7 & 1,69 & 0,078 & 25,9 & 87,1 & 29,9 \\
\hline & 32 & 50,1 & 9,0 & 1,87 & 211,0 & 149,8 & 1,41 & 0,060 & 24,2 & 85,5 & 19,9 \\
\hline & 40 & 49,2 & 8,9 & 1,90 & 215,1 & 161,4 & 1,16 & 0,046 & 23,5 & 85,2 & 16,3 \\
\hline
\end{tabular}

* Cf. : tableau 2. 
important. Dans le cas des laits ultrafiltrés, nous observons par contre une élévation nette des teneurs en matières sèches et azotées et une diminution de la teneur en calcium ainsi que des différents rendements dans ces mêmes conditions.

\section{DISCUSSION}

L'acidification jusqu'au point isoélectrique de laits reconstitués à un taux normal à différentes températures (tab. 1) confirme les observations de Fox et Ernstrom, 1969, qui montrent qu'à $0^{\circ} \mathrm{C}$ la caséine ne précipite pas. La quantité relativement importante de calcium qui reste fixée sur les caséines doit permettre le maintien d'un certain nombre de liaisons intermoléculaires assurant la persistance de la stabilité de la suspension. L'addition d'acide lactique à température ambiante $\left(25^{\circ} \mathrm{C}\right)$ aboutit par contre à la déminéralisation complète et à la précipitation des caséines; mais le phénomène est réversible par refroidissement à $0^{\circ} \mathrm{C}$ et agitation énergique pendant une durée suffisante qui provoquent la fluidisation du mélange «précipité-sérum ». Le retour d'une partie du calcium de la phase soluble vers la phase colloïdale et l'hydratation des caséines qui se produisent alors, sont sans doute à l'origine de ce comportement thixotropique du caillé. Le pourcentage de calcium repassant à l'état colloïdal reste cependant inférieur à celui du lait directement acidifié à basse température et explique la plus grande viscosité et la moindre stabilité des suspensions obtenues. Cette déficience de la refixation du calcium est peut-être liée à l'insolubilisation d'une partie de celui-ci, sous forme de phosphate tricalcique difficile à dissocier par la suite, au moment de l'acidification. Cette hypothèse semble d'ailleurs confirmée par l'enrichissement du lait en calcium que favorise sa refixation sur les protéines et permet d'augmenter la fluidité du produit final.

L'acidification à basse température des différents types de laits et en particulier des laits concentrés (fig. 1) confirme les résultats d'Ernstrom, 1964 et 1965, selon lesquels la stabilisation définitive du $\mathrm{pH}$ après une chute rapide en début d'opération n'intervient que très lentement. L'hydratation des protéines à basse température qui masque une grande partie des groupements actifs des molécules de caséine, comme l'ont montré Alais et Tarodo de la Fuente, 1970, lesquels ne peuvent intervenir alors que très lentement dans la phase finale de l'acidification, rend compte vraisemblablement de ce comportement.

Dans les laits concentrés par évaporation, l'augmentation de taille des édifices micellaires qui rend inaccessible une fraction des groupements actifs et le déplacement des équilibres salins vers la phase colloïdale avec en particulier la fixation de phosphate de 
calcium sur les caséines comme l'indiquent Alais et Tarodo de la Fuente, 1970, rendent compte du $\mathrm{pH}$ initial inférieur $(6,4)$ et du pouvoir tampon plus faible.

Dans le cas des laits concentrés par ultrafiltration, la diminution relative de la force ionique par rapport à la teneur en protéines est responsable de la baisse du pouvoir tampon.

Ces observations montrent qu'il est indispensable d'ajouter l'acide très lentement et sous forte agitation pour obtenir un produit final homogène et d'acidité suffisante.

La composition des laits secs préacidifiés indiquée dans le tableau 2 appelle les remarques suivantes : l'addition de calcium en améliorant la fluidité des laits de fabrications, comme nous l'avons vu précédemment (tab. 1), permet une meilleure efficacité du traitement d'atomisation et conduit en conséquence à des poudres moins hydratées; lors de l'ultrafiltration une partie du lactose et des sels, et en particulier le calcium soluble, est éliminée dans le perméat et le rétentat de lait concentré est donc relativement plus riche en matières azotées, ce qui explique la faiblesse du rapport $\mathrm{Ca} / \mathrm{N}$ par rapport aux laits obtenus par évaporation.

Les caractéristiques des divers types de laits préacidifiés reconstitués (tab. 2) découlent de nos observations précédentes concernant l'influence du mode de concentration sur le pouvoir tampon et de l'effet du séchage sur l'élévation du $\mathrm{pH}$ dû à la disparition d'une partie de l'acide lactique déjà mentionné par Hall et Hedrick, 1971. En effet, les laits concentrés par ultrafiltration du fait de leur meilleur pouvoir tampon nécessitent une addition plus importante d'acide et conduisent pour un même taux de reconstitution à des laits de $\mathrm{pH}$ plus bas que dans le cas de la concentration par évaporation.

En outre l'effet constant provoqué par le séchage est d'autant moins sensible que la quantité d'acide rajoutée au lait de départ est relativement forte par rapport à la matière azotée initiale. De même, l'acidité des laits enrichis en calcium et reconstitués est plus faible car ils ont nécessité à l'origine moins d'acide pour leur préparation (cf. § III 2). Le pourcentage plus important de calcium fixé sur les protéines des laits concentrés par évaporation s'explique par leur rapport $\mathrm{Ca} / \mathrm{N}$ initialement plus élevé que celui des laits ultrafiltrés (tab. 2).

L'influence du taux de reconstitution sur ces mêmes paramètres (tab. 3) est évidente pour le $\mathrm{pH}$ dont la diminution est directement liée à l'augmentation de matières sèches; la fixation accrue du calcium malgré la baisse du $\mathrm{pH}$ résulte sans doute de l'augmentation de la force ionique dans la phase soluble et elle est très sensible dans le cas des laits concentrés par évaporation où cette variation est plus importante. 
La méthode thrombélastographique nous a permis d'évaluer l'aptitude à la floculation et à la coagulation des différents types de laits préacidifiés reconstitués (tab. 4).

La floculation et la coagulation plus rapides observées avec les laits ultrafiltrés tient d'une part à leur plus forte acidité à la reconstitution (tab. 2) comme l'ont signalé Tarodo de la Fuente et Alais, 1975, et d'autre part au mode de concentration lui-même qui favorise l'augmentation relative de la teneur en matières azotées, responsable de la floculation, par rapport à celle du calcium insoluble qui assure leur stabilité (tab. 2) et qui limite la variation du $\mathrm{pH}$ isoélectrique. Ce dernier point rend compte de l'accroissement des temps de floculation et de raffermissement des gels observé avec des laits enrichis en calcium pour lesquels il y a une fixation protéinique plus importante de ce sel à basse température (tab. 2). L'instabilité des laits emprésurés tient évidemment à l'action spécifique de l'enzyme sur la caséine $\mathrm{K}$ et elle a été particulièrement mise en évidence dans le cas des laits acidifiés par Fox et Ernstrom, 1969, et Cheryan et al., 1975.

L'influence du taux de reconstitution s'exerce différemment sur la floculation et sur la coagulation (tab. 5). La chute de la vitesse de floculation avec l'augmentation de la concentration des matières sèches tient à la plus grande stabilité des protéines résultant de l'accroissement parallèle du taux de calcium fixé (tab. 3). En revanche lorsque la floculation a eu lieu, le rapprochement des protéines consécutif à leur concentration accélère la vitesse de formation du gel comme Roongtanapirom et al., 1979, l'ont déjà indiqué. Dans tous les cas, les variations inverses des temps de floculation et de coagulation se compensent et conduisent à des températures finales voisines pour un même mode de concentration du lait.

Nous retrouvons un effet comparable des différents paramètres étudiés sur la synérèse et l'aspect des coagulums des laits préacidifiés reconstitués (tab. 6,7 et 8 ) avec en particulier des variations dans le même sens de la vitesse d'égouttage et de floculation.

La description du mécanisme de la synérèse et des principaux facteurs agissant sur elle par Tarodo de la Fuente, 1971, permet d'interpréter la majeure partie des observations réalisées : c'est ainsi que les protéines, responsables essentielles de la formation du gel et d'une importante rétention d'eau sont à l'origine, dans les laits ultrafiltrés où elles sont relativement concentrées, d'un égouttage plus lent et moins prononcé aboutissant cependant à des coagulums plus fermes que ceux des laits évaporés (tab. 6).

L'action du calcium sur le ralentissement de la synérèse est également attendue ; par contre ses effets faibles et parfois contradictoires sur les quantités de sérum libéré et la fermeté des coagulums (tab, 6) résultent peut-être des variations de répartition de ce sel entre la 
phase aqueuse et la phase colloïdale ; dans nos précédents travaux, Roongtanapirom et al., 1979, montrent en effet que suivant que le calcium ajouté se retrouve en majorité sous forme soluble ou insoluble, l'égouttage est accéléré ou freiné.

L'emprésurage, en augmentant la vitesse de la synérèse et le volume de sérum exsudé, grâce à la libération du macropeptide hydrophile et à la diminution de la charge des caséines qui accroît leur instabilité, conduit à des gels fermes (tab. 6).

L'augmentation du taux de reconstitution et donc de la teneur relative en matières sèches se traduit évidemment dans le tableau 7 par un égouttage ralenti et réduit et un coagulum compact.

Enfin, les coefficients de température des réactions intervenant dans la coagulation étant en général importants, l'élévation de la température de réchauffement ne peut qu'entraîner une accélération de l'ensemble du processus avec pour conséquence une amélioration de la fermeté des produits finaux comme le montre le tableau 8.

Les compositions chimiques des sérums et des caillés en relation avec les différents facteurs mis en jeu (tab. 9, 10 et 11) sont interdépendantes.

Certaines tendances peuvent être soulignées comme par exemple la richesse relative en matières azotées et en calcium des caillés provenant des laits ultrafiltrés qui tient à leur mode de concentration avec cependant un rapport $\mathrm{Ca} / \mathrm{N}$ inférieur dû à la fuite d'une partie des sels dans le perméat (tab. 9) ; l'accroissement en calcium et de l'extrait sec en général des caillés de laits enrichis en cet élément (tab. 9) signalé par Tarodo de la Fuente et al., 1971 ; l'augmentation du taux de matières sèches du caillé avec la température de chauffage (tab. 11) qui confirme également les résultats obtenus à partir des laits écrémés directement acidifiés par Shehata et Olson, 1976 ; et enfin la valeur élevée dans l'ensemble du rapport $\mathrm{Ca} / \mathrm{N}$ qui traduit une minéralisation importante des caillés acidifiés à basse température déjà mise en évidence par Fox et Ernstrom, 1969.

L'élévation de l'extrait sec des caillés avec le taux de reconstitution semble normale (tab. 10), mais il est à remarquer que la teneur en matières azotées est loin de suivre la même évolution.

La fermeté des caillés (tab. 6, 7 et 8) définie comme le rapport des extraits secs (Re) des caillés et des sérums (cf. § II), est directement liée à leurs compositions (tab. 9, 10 et 11), et doit donc être supérieure dans le cas des laits ultrafiltrés étant donné leurs teneurs plus élevées en matières sèches et en protéines et la faiblesse de l'extrait sec des sérums correspondants.

L'effet de l'ajout de calcium différent suivant le mode de concentration est à rapprocher de celui précédemment signalé à propos de 
la synérèse et qui dépend de la répartition de ce sel entre les phases aqueuse et colloïdale du lait ; l'augmentation du rapport $\mathrm{Ca}$ fixé/Ca soluble dans le lait ultrafiltré compte tenu de la fuite des sels dans le perméat, peut expliquer la réduction de l'égouttage et la fermeté du caillé. La diminution de ce même rapport dans le lait évaporé enrichi en calcium rend compte inversement d'une exsudation plus poussée et d'une meilleure fermeté du caillé qui a déjà été observée avec des laits concentrés par ce procédé et acidifiés directement par Tarodo de la Fuente et al., 1971.

Ernstrom, 1964, a montré que l'emprésurage à basse température des laits préalablement acidifiés conduit à des caillés mieux égouttés et donc plus fermes et nous le constatons aussi quel que soit le type de concentration.

La chute de la fermeté lors de l'accroissement du taux de reconstitution traduit l'augmentation relativement plus forte de l'extrait sec des sérums par rapport aux caillés et résulte certainement de l'absence de variation du taux des matières azotées dans le caillé mentionnée ci-dessus (tab. 10).

Lorsque la température de chauffage s'élève, seules les matières sèches du caillé augmentent, ce qui explique la plus grande fermeté de ces derniers comme l'a indiqué Ernstrom, 1964 et 1965, à diverses reprises.

Les rendements en matières azotées, en calcium et surtout fromager (tab. 9, 10, et 11) qui comptent parmi les paramètres les plus intéressants, sont également en étroite relation avec la quantité et la constitution des sérums éliminés lors de l'égouttage; à titre d'exemple : lorsque le volume de sérum diminue avec une teneur en élément identique ou plus faible, il s'ensuit une augmentation du rendement de cet élément dans le caillé et vice versa.

Le procédé de concentration semble important, notamment pour ce qui est des additions de calcium et de présure qui ont des effets opposés puisqu'elles provoquent l'augmentation des rendements avec les laits ultrafitrés et leur diminution avec ceux qui ont été évaporés. Le rendement élevé en matières azotées observé avec les laits ultrafiltrés tient évidemment à la technique de concentration. Ces résultats, en apparence contradictoires et en partie observés par Tarodo de la Fuente et al., 1971, sur des laits évaporés, acidifiés par voie chimique et enrichis en calcium, ont certainement pour origine la répartition différente de ce sel entre les phases aqueuse et colloïdale mentionnées précédemment.

L'amélioration générale des rendements obtenue par l'accroissement de la teneur en matières sèches a déjà été constatée par Roongtanapirom et al., 1979, avec des laits préemprésurés à basse température, séchés et reconstitués à des taux comparables. 


\section{CONCLUSION}

Nos travaux montrent que l'acidification par voie chimique à basse température des laits leur permet de conserver une fluidité suffisante pour qu'ils puissent être séchés.

Les poudres ainsi préparées, par reconstitution et réchauffement conduisent à des caillés de même type que ceux obtenus lors de la fabrication des fromages frais par acidification directe du lait (Cottage Cheese).

Nous avons montré aussi que la déminéralisation et la précipitation des caséines provoquées par l'acidification à température ambiante sont des phénomènes réversibles puisque le refroidissement et l'agitation énergique du mélange « précipité-sérum » permettent la remise en suspension des protéines et la fluidisation de l'ensemble.

Cette propriété peut en partie résoudre le problème posé par l'obligation d'utiliser la voie biologique pour l'acidification des laits destinés à la fabrication des fromages frais dans certains pays dont le nôtre.

Cette voie a d'ailleurs été déjà en partie exploitée dans certains pays orientaux où des poudres de laits coagulés par fermentation lactique conduisant par addition d'eau à des produits de type yaourt (Leben) sont fabriquées suivant un procédé breveté par Ibrahim, 1976.

Lorsque la législation l'autorise il est possible également d'envisager une acidification mixte, d'abord par les bactéries qui apporteraient les flaveurs naturelles des fromages frais, ensuite par voie chimique à basse température qui permettrait de gagner du temps et de régulariser la fabrication.

Nous avons mis en évidence également l'influence de plusieurs facteurs tels que le mode de concentration, l'addition de calcium et de présure, le taux de reconstitution et la température de coagulation sur les caractéristiques physicochimiques des poudres et sur la formation, la composition et la texture des caillés auxquels elles conduisent ; les variations des rendements qui en résultent et qui représentent une notion particulièrement intéressante pour les utilisateurs industriels, ont été résumées dans le tableau synoptique suivant.

Nous constatons qu'avec les laits concentrés par évaporation (EV) seule l'augmentation du taux de reconstitution conduit à de meilleurs rendements, alors qu'avec les laits concentrés par ultrafiltration (UF), cette amélioration est obtenue également par addition de calcium et de présure.

Enfin, nous rappellerons l'intérêt économique et nutritionnel de ces laits secs préacidifiés qui se conservent bien, sont faciles et bon marché à transporter ainsi qu'à mettre en œuvre et permettent, en jouant sur les divers facteurs étudiés et en particulier sur le taux 


\begin{tabular}{|c|c|c|c|c|c|c|}
\hline \multirow{3}{*}{ Facteurs } & \multicolumn{6}{|c|}{ Rendement } \\
\hline & \multicolumn{2}{|c|}{ Fromager } & \multicolumn{2}{|c|}{ Mat } & \multicolumn{2}{|c|}{$\mathrm{Ca}$} \\
\hline & EV & UF & $\mathrm{EV}$ & UF & EV & UF \\
\hline Addition calcium & $\Downarrow$ & $\not$ & $\searrow$ & $\pi$ & $\searrow$ & $\not$ \\
\hline Addition présure & $\mathbb{\Downarrow}$ & $\not$ & $\searrow$ & $\searrow$ & $\searrow$ & $\pi$ \\
\hline $\begin{array}{l}\text { Augmentation MS } \\
\text { (taux de reconstitution) }\end{array}$ & $\not$ & $\not$ & $D$ & $\not T$ & $\not{T}$ & $\not 7$ \\
\hline $\begin{array}{l}\text { Augmentation } \\
\text { température coagulation }\end{array}$ & $\searrow$ & $\searrow$ & $\searrow$ & $\searrow$ & $\searrow$ & $\Downarrow$ \\
\hline
\end{tabular}

$\nearrow$ ou $\searrow$ tendance à l'augmentation ou diminution.

ou $\Downarrow$ augmentation ou diminution nette.

de reconstitution, de réduire l'égouttage, ce qui limite la pollution et améliore le rendement fromager et la valeur nutritionnelle des produits finis.

Reçu pour publication en mars 1982.

\section{Bibliographie}

AlaIs (C.) (1962). - Etude de l'action enzymatique de la présure sur la caséine. Isolement et composition du caséino-glycopeptide. Thèse de Docteur-Ingénieur, Université de Paris.

Alais (C.) and TARodo de La Fuente (B.) (1970). - Effects of milk concentration on the salt equilibria. Milchwissenschaft, 25, 514.

BerRidge (N. J.) (1952). - Some observation on the determination of the activity of rennet. Analyst, 77, 57.

Breene (W. M.) Price (W. V.) and Ernstrom (C. A.) (1964). - Changes in composition of "Cheddar curd" during manufacture as a guide to cheese making by direct acidification. J. Dairy Sci., 47, 840 .

Cheryan (M.), van WYK (P. J.), Olson (N. F.) and Ricardson (T.) (1975). - Secondary phase and mechanism of enzymatic milk coagulation. J. Dairy Sc., $58,477$.

Davies (D. T.) and White (J.C. D.) (1960). - The use of ultrafiltration and dialysis isolation the aqueous phase of milk in determining the partion of milk constituents between the aqueous and disperse phase. J. Dairy Res., $27,171$. 
ERnstrom (C. A.) (1964). - Continuous "Cottage Cheese» manufacture by acidification of low temperature. Milk Dealer, 54, 50.

Ernstrom (C. A.) (1965), - Continuous «Cottage Cheese» manufacture without culture. Milk Ind., 56, 36.

ERnstrom (C.A.) (1965). - Mechanized «Pizza Cheese» making by means of continuous direct acidification method. Mfg. Milk Prod. J., 57, 7.

Fox (C. W.) and ERnstrom (C. A.) (1969). - The role of calcium in the low-temperature acidification of skim milk and the formation of direct-acid curd. J. Dairy Sci., 52, 885.

FrENTZ (R.) (1965). - Application de la thrombélastographie de Hartert à l'étude de la coagulation du lait. Le Lait, 45, 489.

GoRdon (W. G.) and Kalan (E. B.) (1974), - Proteins of milk. In «Fundamentals of Dairy Chemistry", edited by Webb B. H., Johnson A. H. and Alford J.A. The AVI Publishing Company Inc., Westport.

Hall (C. W.) and Hedrick (T. I.) (1971). - Processing, packaging and storage of nonfat dry milk and dry whole milk. In "Drying of milk products», edited by Hall C. W. and Hedrick T. I. The AVI Publishing Company Inc., Westport.

IbRAHIM (I. E.) (1976). - Procédé de préparation de lait fermenté sous forme de poudre ou de granules. Brevet d'invention n ${ }^{\circ} 2345083$ du 23-3-1976 et additif $\mathrm{n}^{\circ} 2397156$ du 12-7-1977.

Larson (W. A.), Olson (N. F.) and Lund (D. B.) (1970). - Continuous direct acidification system for producing «Mozzarella Cheese». J. Dairy Sci., 53, 646.

LELIÈVRE (J.) (1977). - Rigidity modulus as a factor influencing the syneresis of renneted milk gels. J. Dairy Res., 44, 611.

Linden (G.) (1971). - Applications de la spectrophotométrie d'absorption atomique dans les laboratoires d'industrie alimentaire. Ind. Alim. et Agric., 88, 793.

LitTle (L.) (1967). - Techniques for acidified dairy products. J. Dairy Sci., 50, 434.

Roongtanapirom (S.), Bennasar (M.) et Tarodo de la Fuente (B) (1979). - Aptitude à la coagulation de laits préemprésurés secs. Le Lait, 59, 571.

Shemata (A. E.) and Olson (N.F.) (1966). - Manufacture of "Blue Cheese» by direct acidification method. J. Dairy Sci., 49, 1025.

Tarodo de la Fuente (B.) (1971). - Physico-chimie de la séparation du caillé et du sérum, La Technique Laitière, 719, 79.

Tarodo de la Fuente (B.) and Alais (C.) (1975). - Physico-chemical studies on the enzymatic coagulation of milk. Chimia, 29, 379.

Tarodo de la Fuente (B.), Alais (C.) et Frentz (R.) (1969). - Etude de la coagulation du lait par la présure et de la synérèse du coagulum par la méthode thrombélastographique. Le Lait, 49, 400.

Tarodo de la Fuente (B.), Turk (S.), Alais (C.), Hutin (G.) Bruneau (J. C.) (1971). - Influence du procédé de maturation sur les propriétés et la composition des caillés lactiques obtenus par coagulation du lait concentré. Le Lait, 51, 623. 